\title{
Socioeconomic Differences in Social Information Processing and Cardiovascular Reactivity
}

\author{
EDITH CHEN ${ }^{a}$ AND KAREN A. MATTHEWS
}

Department of Psychiatry, University of Pittsburgh School of Medicine, 3811 O'Hara Street, Pittsburgh, Pennsylvania 15213, USA

Low socioeconomic status (SES) has a profound influence on children's physical health. As SES decreases, all-cause mortality rates increase linearly, ${ }^{1}$ and rates of injury, pneumonia, and cancer mortality increase. ${ }^{2}$ One potential explanation for the SES and health relationship may involve social information processing. Lower SES children, because of their more frequent exposure to unpredictable and stressful situations, may develop a schema about the world being a threatening place that requires constant vigilance. This schema may predispose children to interpreting a wide range of situations, including those that are ambiguous in outcome, as threatening. Children who display a bias toward interpreting ambiguous situations as threatening may also be prone to heightened cardiovascular reactivity during such situations. Over time, more frequent episodes of reactivity may place these children at risk for negative health outcomes such as cardiovascular disease (See FIG. 1).

Research has demonstrated that lower SES is associated with heightened reactivity to laboratory stressors ${ }^{3}$ and higher resting levels of blood pressure ${ }^{4}$ among children. Trait variables such as hostility mediate this relationship among AfricanAmerican children. ${ }^{3}$ In addition, adults in lower status occupations who also have hostile attributional styles have higher blood pressure elevations at work. ${ }^{5}$ However, the role of situationally based variables such as social information-processing biases has not been investigated in children.

We tested the mediational role of social information processing in a sample of 198 children (ages 8-17), half Caucasian and half African-American (Time 1: T1). Ninety of these children were retested an average of three years later (Time 2: T2). At both times, children were probed about perceptions of hostile intent and anger in response to scenarios with negative or ambiguous outcomes (Social Cognition Interview). At both times, cardiovascular reactivity was averaged across three laboratory stress tasks (mirror tracing, reaction time, cold forehead). SES was measured by family and paternal Hollingshead scores (based on parent education and occupation).

At T1, among Caucasians, low-SES children responded with more hostile intent perceptions and anger than high-SES children during ambiguous, but not negative, scenarios (see TABLE 1). In addition, analyses of the reactivity data revealed that among Caucasians, lower SES was associated with greater vascular reactivity

\footnotetext{
${ }^{a}$ Address for correspondence: Edith Chen, Ph.D., University of Pittsburgh School of Medicine, 3811 O'Hara St., Pittsburgh, PA 15213, USA. 412-624-0946 (voice); 412-624-0967 (fax). e-mail: chene@msx.upmc.edu
} 


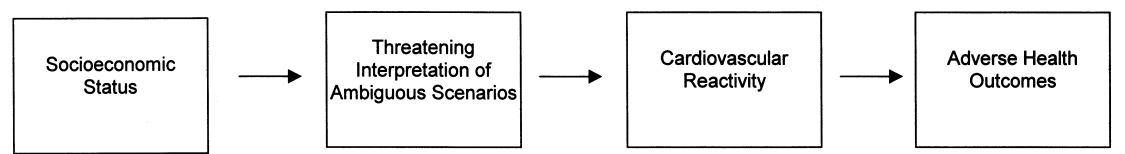

FIGURE 1.

TABLE 1. Correlations between SES and Social Cognition Interview responses by race

\begin{tabular}{|c|c|c|}
\hline & Caucasian & $\overline{\text { African American }}$ \\
\hline \multicolumn{3}{|c|}{ Ambiguous scenarios (Time 1) } \\
\hline Hostile intent & $-0.27^{a}$ & -0.13 \\
\hline Anger & $-0.23^{b}$ & -0.19 \\
\hline \multicolumn{3}{|c|}{ Negative scenarios (Time 1) } \\
\hline Hostile intent & 0.00 & -0.05 \\
\hline Anger & 0.18 & -0.15 \\
\hline \multicolumn{3}{|c|}{ Ambiguous scenarios (Time 2) } \\
\hline Hostile intent & 0.07 & $-0.57^{a}$ \\
\hline Anger & $-0.33^{b}$ & -0.21 \\
\hline \multicolumn{3}{|c|}{ Negative scenarios (Time 2) } \\
\hline Hostile intent & -0.13 & -0.19 \\
\hline Anger & 0.23 & -0.26 \\
\hline \multicolumn{3}{|c|}{ Ambiguous scenarios (Time 2 - Time 1 ) } \\
\hline Hostile intent & 0.19 & $-0.49^{a}$ \\
\hline Anger & 0.26 & -0.01 \\
\hline \multicolumn{3}{|c|}{ Negative scenarios (Time 2 - Time 1 ) } \\
\hline Hostile intent & -0.03 & -0.12 \\
\hline Anger & 0.05 & -0.18 \\
\hline \multicolumn{3}{|c|}{ Ambiguous scenarios (Time $1+$ Time 2 ) } \\
\hline Hostile intent & -0.06 & $-0.55^{a}$ \\
\hline Anger & 0.16 & $-0.37^{b}$ \\
\hline \multicolumn{3}{|c|}{ Negative scenarios (Time $1+$ Time 2 ) } \\
\hline Hostile intent & -0.03 & -0.07 \\
\hline Anger & $0.33^{\mathrm{b}}$ & $-0.45^{b}$ \\
\hline
\end{tabular}

NoтE: Higher scores reflect higher social class, and greater perceptions of hostile intent or anger. ${ }^{a} p<0.01$.

${ }^{b} p<0.05$. 
[greater increases in diastolic blood pressure (DBP) and total peripheral resistance (TPR)] in response to laboratory stressors. When response to ambiguous scenarios was partialled out, the percent of variance in DBP/TPR reactivity that SES accounted for decreased by $18-43 \%$.

At T2, among African Americans, lower SES was associated with increases in systolic blood pressure (SBP) in response to laboratory stressors. In addition, lower SES was associated with greater perceptions of hostile intent during ambiguous scenarios at T2 (see TABLE 1). Finally, when response to ambiguous scenarios was partialled out, the percent of variance in SBP reactivity that SES accounted for decreased by $31 \%$. In addition, longitudinal analyses revealed that among African Americans, lower SES at T1 was associated with both increases in hostile intent perceptions from $\mathrm{T} 1$ to $\mathrm{T} 2$ and with consistently high hostile intent perceptions at both T1 and T2 (see TABLE 1). When increases in hostile intent perceptions were partialled out, the percent of variance in T2 SBP reactivity that SES accounted for decreased by $70 \%$. When consistently high hostile intent perceptions were partialled out, the percent of variance in T2 SBP reactivity that SES accounted for decreased by $28 \%$.

In sum, social information-processing biases appears to be a promising mediator explaining the SES and cardiovascular reactivity relationship in children. Although SES contributes only a small amount to the variance in cardiovascular reactivity, social information processing biases explain a substantial portion of these SES effects.

\section{REFERENCES}

1. Mare, R.D. 1982. Socioeconomic effects on child mortality in the United States. Am. J. Public Health 72: 539-547.

2. Nelson, M.D. 1992. Socioeconomic status and childhood mortality in North Carolina. Am. J. Public Health 82: 1131-1133.

3. Gump, B.B., K.A. Matthews \& K. RaikKonen. 1999. Modeling relationships among socioeconomic status, hostility, cardiovascular reactivity, and left ventricular mass in African-American and White children. Health Psychol. 18: 140-150.

4. Wright, L.B., F. Treiber, H. Davis, C. Bunch \& W.B. Strong. 1998. The role of maternal hostility and family environment upon cardiovascular functioning among youth two years later: socioeconomic and ethnic differences. Ethn. Dis. 8: 367-376.

5. Flory, J.D., K.A. MatThews \& J.F. Owens. 1998. A social information processing approach to dispositional hostility: relationships with negative mood and blood pressure elevations at work. J. Soc. Clin. Psychol. 17: 491-504. 